Rev. Saúde públ., S. Paulo

7:103-13, 1973.

\title{
QUANTIFICAÇÃO DO INDICADOR DE NELSON DE MORAES (CURVA DE MORTALIDADE PROPORCIONAL)
}

\author{
José da SiIva GUEDES * \\ Marilda Lauretti da Silva GUEDES *
}

RSPU-B/164

\begin{abstract}
Guedes J. DA S. \& Guedes M. L. DA S. - Quantificação do indicador de Nelson de Moraes (curva de mortalidade proporcional). Rev. Saúde públ., S. Paulo, 7:103-13, 1973.

RESUMO: Propõe-se um critério para quantificação do indicador de Nelson de Moraes (curva de mortalidade proporcional) procurando eliminar as desvantagens do indicador não ter tradução numérica. Utilizando dados de Moraes, Ramos e próprios, são quantificadas Curvas de Mortalidade Proporcional de países desenvolvidos, do municipio de São Paulo e das regiões administrativas do Estado de São Paulo. A quantificação gera um indicador cujas cifras variam de valores negativos até o máximo teórico de +50 . São destacadas as vantagens da quantificação, que permite a hierarquização de diferentes regiôes e a comparação simultânea da evoluçấo do nivel de saúde de um grande número de localidades.
\end{abstract}

UnItermos: Niveis de saúde (indicadores) *; Estatística vital *; Mortalidade*; Epidemiologia.

\section{N T R O D U C A O}

Em 1952, a ONU (apud Swaroop \& Uemura ${ }^{6}$ ) convocou um Comitê de Peritos com o fim de elaborar a maneira mais adequada para medir os "níveis de vida", que sugeriu uma série de doze componentes, figurando dentre eles "saúde, incluindo condições demográficas".

O primeiro indicador criado para medir "saúde, incluindo condições demográficas" foi o de Swaroop \& Uemura ${ }^{6}$, a Razão de Mortalidade Proporcional, tendo sido apresentado em 1955 ao Grupo de Estudo sobre a medida do nível de saú- de, que sobre o patrocínio da OMS se reuniu em Genebra.

Em 1957, as conclusões do citado Grupo de Estudo 4 foram publicadas incluindo o indicador de Swaroop e Uemura dentre os indicadores globais de saúde e sugerindo que fossem feitos estudos sobre a mortalidade proporcional em determinadas idades (outras que a de 50 anos e mais).

A partir dessa sugestão, Morais ${ }^{3}$, em 1959 , estudando a mortalidade proporcional para as idades: menores de 1 ano, 1

Do Departamento de Medicina Social da Faculdade de Ciências Médicas da Santa Casa de Sáo Paulo - Rua Cesário Mota Jr., 112 - São Paulo, SP. - Brasil. 
GUEDES, J. da S. \& GUEDES, M. L. da S. - Quantificação de indicador de Nelson de Moraes (curva de mortalidade proporcional). Rev. Saúde públ., S. Paulo, 7:103-13, 1973.

a 4,5 a 19,20 a 49 e 50 anos e mais, propôs um novo indicador, a Curva de Mortalidade Proporcional. Este indicador apresenta uma série de vantagens: dispensa os dados de população, é fácil de calcular, inclui o indicador de Swaroop e Uemura, e além disso, permite visualização da situação pois é representado graficamente.

Vários autores têm utilizado o indicador de Nelson de Moraes para localidades brasileiras 2,5 .

Alguns inconvenientes têm sido sentidos, entretanto, no seu uso, decorrentes primordialmente do fato do indicador não ser expresso numericamente. Assim, para curtos períodos de tempo em que tenha ocorrido pequenas variações, a percepção de diferenças pode não ser muito rápida, já que depende da análise do comportamento de 5 pontos do gráfico e por não haver um critério expresso quanto ao significado do aumento ou diminuição para os diferentes grupos abaixo de 50 anos. A comparação de trabalhos usando gráficos em escalas diferentes também não é fácil. Encontra-se dificuldade em descrever as mudanças quando compreendidas nos intervalos entre os 4 tipos esquemáticos (elevado, regular, baixo e muito baixo). $\mathbf{E}$ finalmente, é impossível atribuir "nota" ou "peso" ao indicador, nas tentativas de reunir vários indicadores para obter uma "nota" média para a situação de saúde de uma localidade.

Procurando estudar a evolução do nível de saúde das regiões administrativas do Estado de São Paulo, de 1950 a 19701 deparamos com o problema de comparar as curvas de mortalidade proporcional das onze regiões durante os períodos de 1949 a 1951, 1959 a 1961, 1966 a 1968. Os gráficos são auto-explicativos, porém, tentar analisar as diferenças entre as curvas das onze regiões, de modo a poder hierarquizá-las, foi algo bastante difícil, o que nos levou a procurar uma "quantificação" do indicador.

\section{MATERIAL E MÉTODOS}

Para o propósito da quantificação do indicador de Nelson de Moraes utilizamos: dados referentes ao município de São Paulo de 1894 a 1959 calculados a cada 5 anos, apresentados por RAMos 5 ; dados referentes a sete países desenvolvidos por volta de 1950, apresentados por Morais ${ }^{3}$ e dados referentes às regiões administrativas do Estado de São Paulo para os períodos de 1949-1951, 1959-1961, 1966-1968, por nós apresentados 1 .

Buscamos estabelecer pesos para cada ponto da curva segundo um raciocínio que levou em conta o "poder discriminatório" da porcentagem de mortes em ca da grupo de idade, que depende em última análise da vulnerabilidade do grupo às alterações das condições de vida.

Assim atribuimos:

a. pontos positivos para a proporção de mortes nas idades acima de $\mathbf{5 0}$ anos, já que seu aumento revela uma melhoria de saúde;

b. pontos negativos para a proporção de mortes nas idades abaixo de $\mathbf{5 0}$ anos, já que seu aumento revela piora do nível de saúde;

c. peso + 5 para a proporção de mortes no grupo de 50 anos e mais;

d. peso - 4 para a proporção de mor tes no grupo de menores de 1 ano. A mortalidade neste grupo reflete bem a proteção oferecida aos infantes contra as agressões do meio, dependendo essa proteção de inúmeros fatores de ordem social, econômica e cultural. É ainda, em geral, o primeiro grupo a sofrer as consequiências das alterações sócio- 
GUEDES, J. da S. \& GUEDES, M. L. da S. - Quantificação do indicador de Nelson de Moraes (curva de mortalidade proporcional). Rev. Saúde pübl., S. Paulo, 7:103-13, 1973.

econômicas de uma comunidade e, por tudo isso, decidimos conferirlhe o maior peso negativo, a fim de que suas alterações pudessem influir seguramente no indicador quantificado.

e. peso - 3 para a proporção de mortes no grupo de 20 a 40 anos, já que, quando sua contribuição para a mortalidade é alta, as condições gerais de saúde são más, pois para grande parte das causas de morte encontradas neste grupo etário há recursos para prevenção e tratamento. Acresce ainda que este grupo já foi "selecionado" nas idades anteriores, devendo ser constituído por indivíduos com melhores condições para sobrevivência, cuja maioria deveria ultrapassar incólume mais esta faixa etária;

f. peso -2 para a proporção de mortes no grupo de 1 a 4 anos de idade, que apresenta valores de mortalidade bem distintos em regiões desenvolvidas e subdesenvolvidas, em função principalmente da frequiência das doenças infecciosas e da desnutrição. Este grupo é menos vulnerável às alterações sócioeconômicas do que o grupo de menores de 1 ano.

g. peso - 1 para a proporção de mortes no grupo de 5 a 19 anos de idade, que tem pequeno poder discriminatório, pois em quase todos os níveis de saúde, do "muito baixo" ao "elevado", apresenta valores percentualmente baixos.

\section{Cálculo do Indicador}

Multiplica-se a porcentagem de óbitos correspondente a cada grupo pelo seu peso, procede-se à soma algébrica dos resultados e divide-se por dez.

A fixação do peso +5 para a propor- ção de mortes no grupo de 50 anos e mais, ao invés de +10 (já que a soma dos pesos negativos é - 10), deve-se a que desse modo o indicador varia desde valores negatvos, até um valor máximo teórico de +50 e as curvas consideradas por Moraes como indicativas de nível de saúde regular apresentam "valores quantificados" em torno de zero.

\section{RESULTADOS E DISCUSSAO}

O cálculo da "quantificação" para os dados do município de São Paulo de 1894 a 1967 (Tabela 1) mostra que a variação dos valores se faz com regularidade e que, dentro da faixa de "nível muito baixo", consegue detectar uma série grande de estágios intermediários, os quais pelo critério "visual" seriam expressos apenas como "transição" de "muito baixo" para "baixo", com grande dificuldade para o estabelecimento de graus. No sentido de avaliar o interesse da quantificação, devem ser cotejados os dados da Tabela 1 com a Figura 1 extraídos do trabalho de RAmos 5 .

Outra vantagem da quantificação das curvas de mortalidade proporcional é permitir-nos a fácil elaboração de gráficos para análise simultânea da evolução do nível de saúde de várias localidades ao longo do tempo, o que pode ser visto comparando-se as Figuras 2, 3, 4, com a 5 e a Tabela 3 .

Como pode ser visto nas Tabelas 1 a 2 há um certo paralelismo entre os valores da curva de mortalidade proporcional quantificada e os do indicador de Swaroop e Uemura. Em estudo posterior calcularemos o poder discriminatório deste indicador quantificado que a nosso ver deve ser alto, já pelo fato de incluir na sua estrutura e com peso grande o indicador de Swaroop e Uemura, o de mais alto poder discriminatório até agora conhecido. 
GUEDES, J. da S. \& GUEDES, M. L. da S. - Quantificaçāo do indicador de Nelson de Moraes (curva de mortalidade proporcional). Rev. Saúde pübl., S. Paulo, 7:103-13, 1973.

T A B E L A 1

Curvas de mortalidade proporcional, quantificação da mortalidade proporcional e indicador de Swaroop-Uemura para o município de São Paulo, de 1894 a 1967.

\begin{tabular}{|c|c|c|c|}
\hline Ano & Tipo de curva & “Quantificação” & Indicador de \\
\hline 1894 & muito balxo & $-20,6$ & 11,4 \\
\hline 1899 & & $-20,4$ & 11,6 \\
\hline 1909 & & $-16,2$ & 16,7 \\
\hline 1914 & & $-18,5$ & 14,0 \\
\hline 1919 & & $-15,9$ & 16,6 \\
\hline 1924 & & $-13,0$ & 20,3 \\
\hline 1929 & baixo & $-12,1$ & 22,5 \\
\hline 1934 & & $-7,4$ & 28,7 \\
\hline 1939 & & $-5,5$ & 30,6 \\
\hline 1944 & regulaı & $-1,4$ & 35,7 \\
\hline 1949 & & $-0,4$ & 38,9 \\
\hline 1954 & & $+1,3$ & 40,7 \\
\hline 1959 & & $+\quad 4,3$ & 44,2 \\
\hline 1967 & & $+\quad 6,5$ & 46,9 \\
\hline
\end{tabular}

FONTE: Dados modificados a partir de RAMOs ${ }^{5}$ e Guedes ${ }^{1}$

$$
\text { T A B E L A } 2
$$

Curvas de mortalidade proporcional, quantificação da mortalidade proporcional e indicador de Swaroop-Uemura para sete países desenvolvidos, por volta de 1950 .

\begin{tabular}{|c|c|c|c|}
\hline País & Tipo de curva & Quantificação & $\begin{array}{c}\text { Indicador } \\
\text { Swaroop-Uemura }\end{array}$ \\
\hline Inglaterra & Nível elevado & 36,3 & 83,1 \\
\hline Suécia & & 36,1 & 82,7 \\
\hline Suiça & & 33,9 & 79,9 \\
\hline Nova Zelândia & & 33,6 & 79,5 \\
\hline Dinamarca & & 33,0 & 79,1 \\
\hline Holanda & & 32,7 & 79,5 \\
\hline E.U.A. & & 29,2 & 74,3 \\
\hline
\end{tabular}

FONTE: Dados modificados a partir de MoraEs ${ }^{3}$. 
GUEDES, J. da S. \& GUEDES, M. L. da S. - Quantificação do indicador de Nelson de Moraes (curva de mortalidade proporcional). Rev. Saúde públ., S. Paulo, 7:103-13, 1973.

T A B E L A 3

Valores da curva de mortalidade proporcional "quantificada" para as regióes administrativas do Estado de São Paulo nos períodos de 1950, 1960 e 1967.

\begin{tabular}{|c|c|c|c|}
\hline Região & 1950 & 1960 & 1967 \\
\hline Grande São Paulo & $-2,1$ & $+\quad 3,6$ & $+\quad 5,2$ \\
\hline Litoral $\quad \ldots \ldots \ldots$ & $-4,0$ & $+\quad 2,2$ & $\begin{array}{l}+5,8 \\
+\quad 5\end{array}$ \\
\hline Vale do Paraíba & $-9,1$ & - 4,4 & $+\quad 2,2$ \\
\hline Sorocaba & $-8,0$ & $-2,0$ & $\begin{array}{r}4,1 \\
+\quad 1\end{array}$ \\
\hline Campinas & $-\quad 1,0$ & $+\quad 5,3$ & $+10,0$ \\
\hline Ribeirão Preto & $-3,7$ & $+\quad 2,6$ & $+8,2$ \\
\hline Bauru $\quad \ldots \ldots$ & $-7,7$ & $+\quad 0,4$ & $+9,0$ \\
\hline S. J. Rio Preto & $-8,4$ & $-3,0$ & $+\quad 3,2$ \\
\hline Araçatuba $\quad \ldots \ldots \ldots$ & $-15,7$ & $-9,6$ & - 2,3 \\
\hline Presidente Prudente & $-21,3$ & $-16,6$ & $-\quad 5,2$ \\
\hline Marilia & $\cdots 13,7$ & $-6,8$ & $+\quad 1,1$ \\
\hline Estado & - 6,8 & - 1,0 & $+5,0$ \\
\hline
\end{tabular}

Fonte dos dados brutos: Deptarmento de Estatística do Estado de S. Paulo.

Para melhor compreensão da maneira como foram feitos os cálculos, apresenta- mos na Tabela 4 os dados referentes ao município de São Paulo.

T A B E L A 4

Percentual de óbitos por grupos de idade no municíplo de Săo Paulo de 1894 a 1967.

\begin{tabular}{|c|c|c|c|c|c|c|}
\hline \multirow{2}{*}{ Ano } & \multicolumn{5}{|c|}{ Grupos de idades } & \multirow{2}{*}{$\begin{array}{c}\text { Quantifi- } \\
\text { cação }\end{array}$} \\
\hline & $<\quad 1$ & $1-4$ & $5-19$ & $20-49$ & $50+$ & \\
\hline 1894 & 33,65 & 20,27 & 7,14 & 26,96 & 11,41 & $-20,6$ \\
\hline 1899 & 34,73 & 22,58 & 6,89 & 23,72 & 11,61 & $-20,4$ \\
\hline 1909 & 32,36 & 22,41 & 6,91 & 21,49 & 16,73 & $-16,2$ \\
\hline 1914 & 34,61 & 18,20 & 9,37 & 23,70 & 14,03 & $-18,5$ \\
\hline 1919 & 30,56 & 22,55 & 7,76 & 22,36 & 16,56 & $-15,9$ \\
\hline 1924 & 29,61 & 19,25 & 6,79 & 22,58 & 20,31 & $-13,0$ \\
\hline 1929 & 30,84 & 17,14 & 5,95 & 23,38 & 22,49 & $-12,1$ \\
\hline 1934 & 28,06 & 11,41 & 6,40 & 25,40 & 28,68 & - 7,4 \\
\hline 1939 & 25,34 & 13,32 & 6,22 & 24,53 & 30,58 & $-\quad 5,5$ \\
\hline 1944 & 22,49 & 10,24 & 6,15 & 25,35 & 35,74 & $-\quad 1,4$ \\
\hline 1949 & 24,33 & 7,89 & 4,51 & 24,34 & 38,87 & $-\quad 0,4$ \\
\hline 1954 & 26,75 & 6,57 & 3,73 & 22,21 & 40,69 & $+\quad 1,3$ \\
\hline 1959 & 24,82 & 6,35 & 3,62 & 21,04 & 44,16 & $+\quad 4,3$ \\
\hline 1967 & $\begin{array}{l}23,67 \\
(-4)\end{array}$ & $\begin{array}{r}4,86 \\
(-2)\end{array}$ & $\begin{array}{r}3,66 \\
(-1)\end{array}$ & $\begin{array}{l}20,77 \\
(-3)\end{array}$ & $\begin{array}{r}47,04 \\
(+5)\end{array}$ & $+6,5$ \\
\hline
\end{tabular}

FONTE: RAMOS $^{5}$ e GUEDES ${ }^{1}$. 
GUEDES, J. da S. \& GUEDES, M. L. da S. - Quantificação do indicador de Nelson de Moraes (curva de mortalidade proporcional). Rev. Saúde públ., S. Paulo, 7:103-13, 1973.

\section{CON CLUSOES}

- A transformação do indicador de Nelson de Moraes com a introdução da quantificação ora proposta origina um indicador de saúde que apresenta cifras variando desde valores negativos até o valor teórico máximo de +50 . As curvas que Moraes classificou como indicativas de nível de saúde regular, apresentam à quantificação valores próximos de zero.
- A quantificação facilita a interpretação das mudanças ocorridas numa mesma localidade e principalmente a comparação simultânea da evolução do nível de saúde em várias localidades, permitindo a elaboração de gráficos.

- A quantificação permite com mais facilidade do que o uso exclusivo dos gráficos, a hierarquização de diferentes localidades quanto ao nível de saúde.

RSPU-B/164

Guedes, J. da S. \& Guedes, M. L. da S. - [Quantification of the Nelson de Moraes's indicator (curve of proportional mortality)]. Rev. Saúde públ,
7:103-13, 1973.

SUMmary: Working with the Nelson de Moraes's indicator (curve of proportional mortality) it is introduced a criterion to quantify the curves, trying in this way a numeric value that, synthetizing the contribution of the different age groups to the mortality, indicates the level of health.

UNITERMS: Health levels (indicators)*; Vital statistics*; Mortality*; Epidemiology.

\section{REFERENCIAS BIBLIOGRAFICAS}

1. GUEDES, J. da S. - Contribuição para o estudo da evolução do nível de saúde do Estado de São Paulo: análise das regióes administrativas (1950-1970). São Paulo, 1972 (Tese Faculdade de Saúde Pública da USP).

2. MASCARENHAS, R. dos S. - Indicadores de saúde para regiōes subdesenvolvidas. Arq. Hig., S. Paulo, 26:291-303, 1961.

3. MORAeS, N. L. de A. - Níveis de saúde de coletividades brasileiras. Rev. Serv. Saúde públ., R. de Janeiro, 10:403-97, 1959.

4. ORGANIZAÇÃO MUNDIAL DA SAÚDE. Study Group of the Measurement of
Levels of Health, Geneva, 1955. Report. Geneva, 1957. (Techn. Rep. Ser., 137).

5. RAMOS, R. - Indicadores do nivel de saúde: sua aplicação ao municipio de S. Paulo (1894-1959). São Paulo, 1962. (Tese - Faculdade de Higiene e Saúde Pública da USP).

6. SWAROOP, S. \& UEMURA, K. - Proportional mortality of 50 years and above: a suggested indicator of the component "health, including demographic conditions" in the measurement of levels of living. Bull. Wld. Hlth. Org., 17:439-81, 1957. 
1894

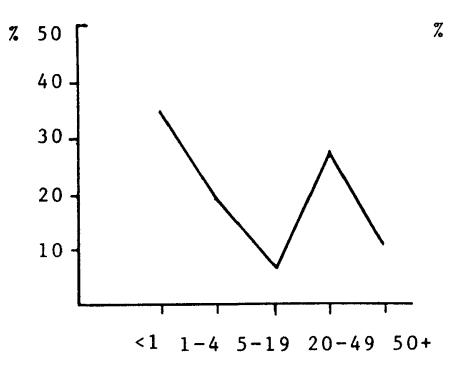

1914

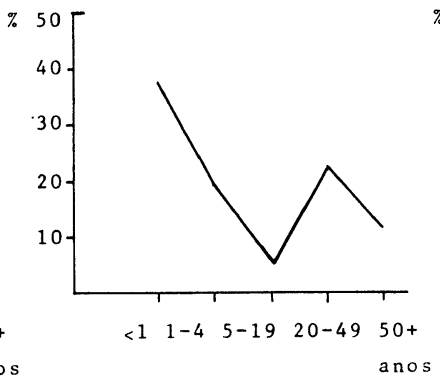

1924

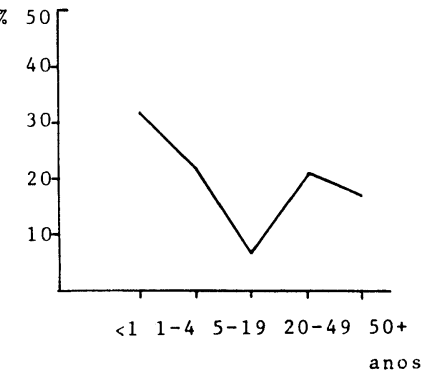

1929

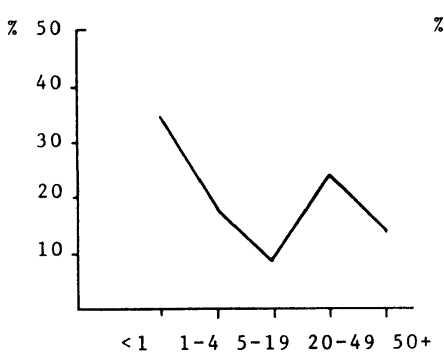

anos

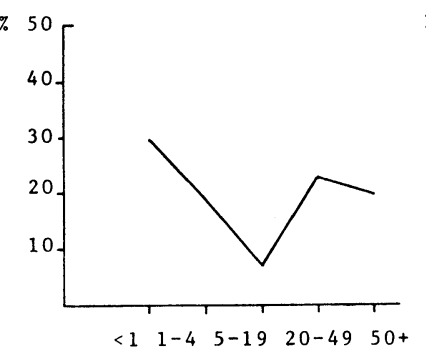

anos

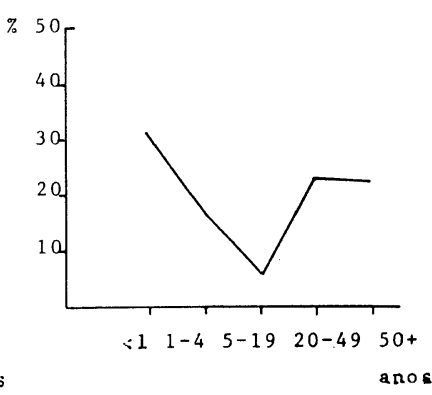

1944

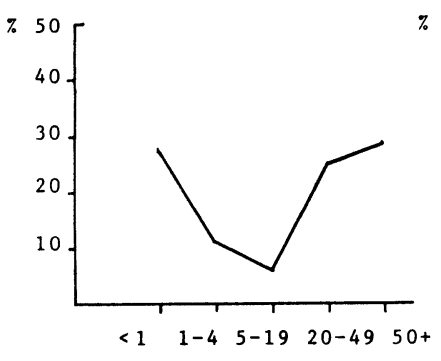

anos

1939

1934

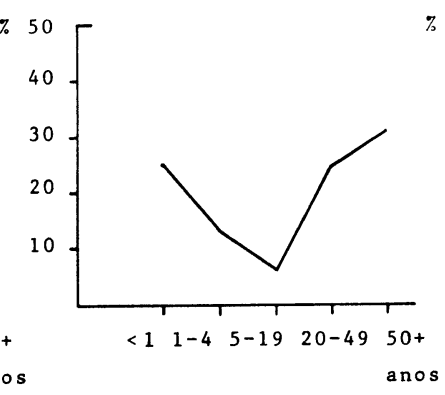

1954

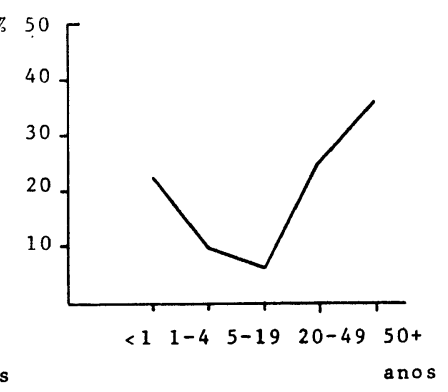

1959
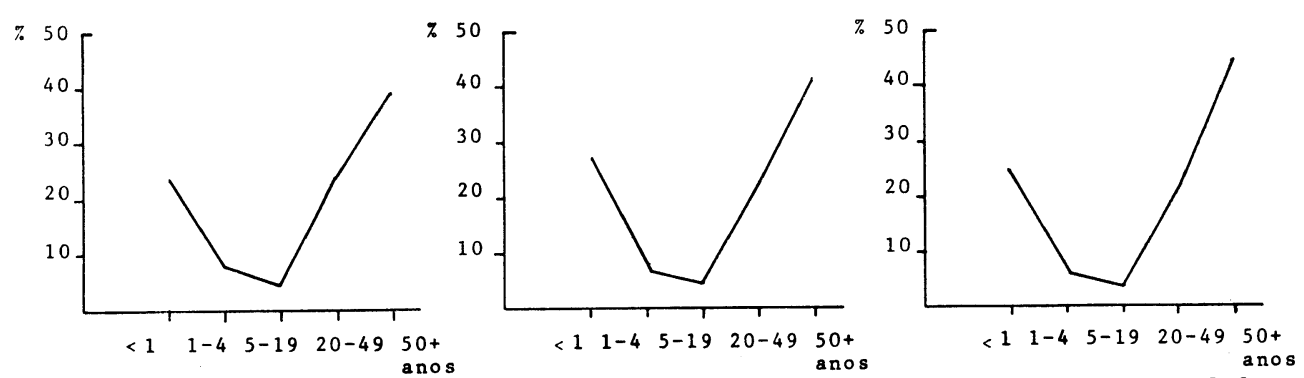

Fig. 1 - Curva de mortalidade proporcional no município de São Paulo. Calculada a cada 5 anos (exceto 1904 e 1919), no período de 1894 e 1959.

Fonte: Ramos 5 modificado. 


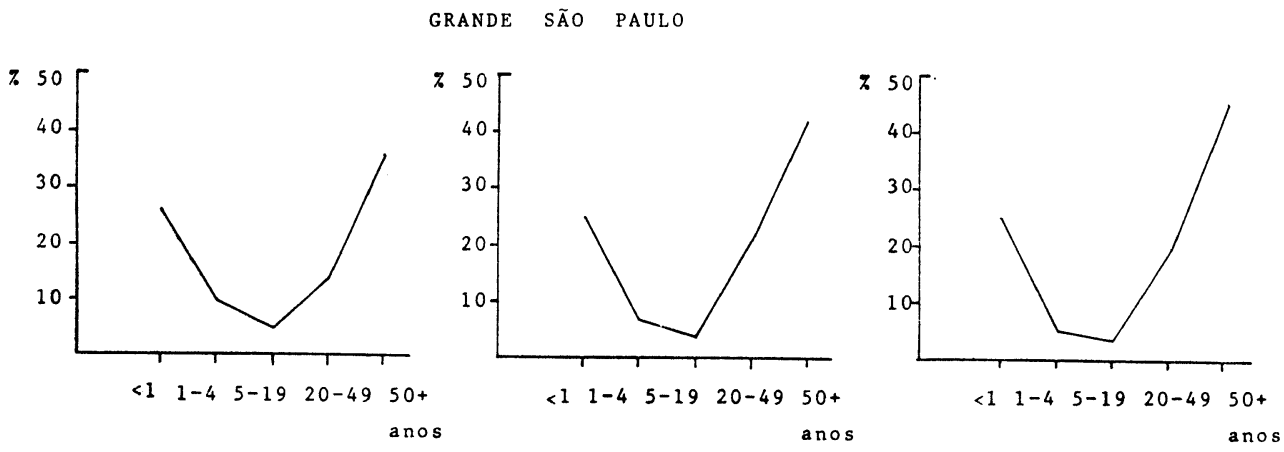

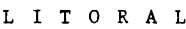
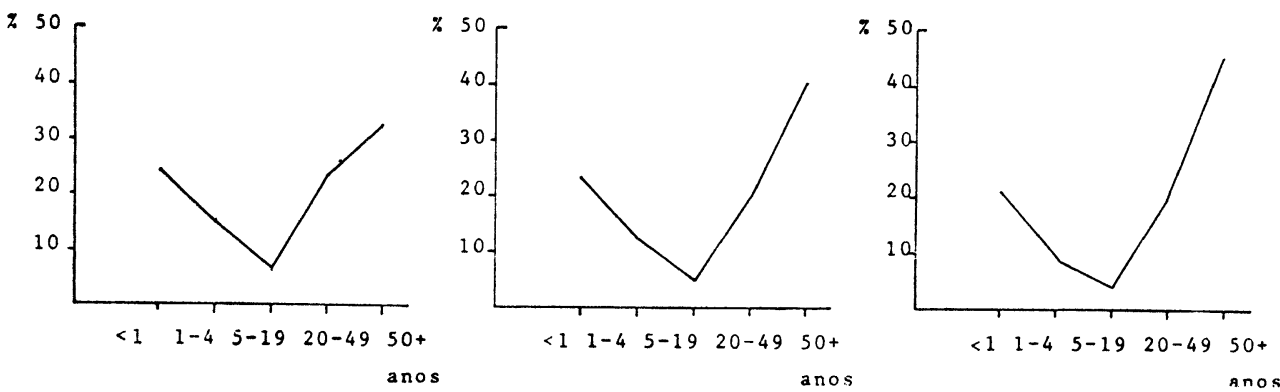

VALE DO PARAIBA
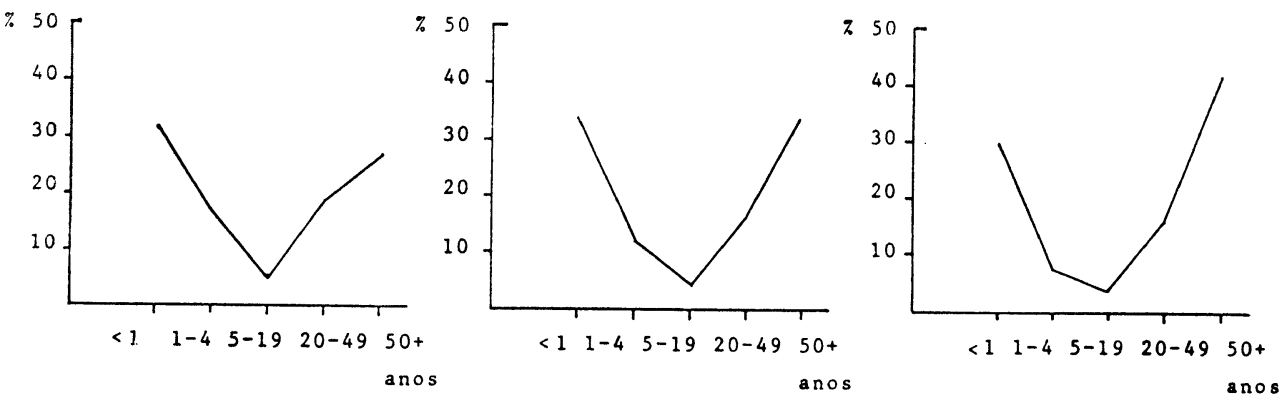

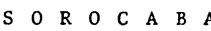
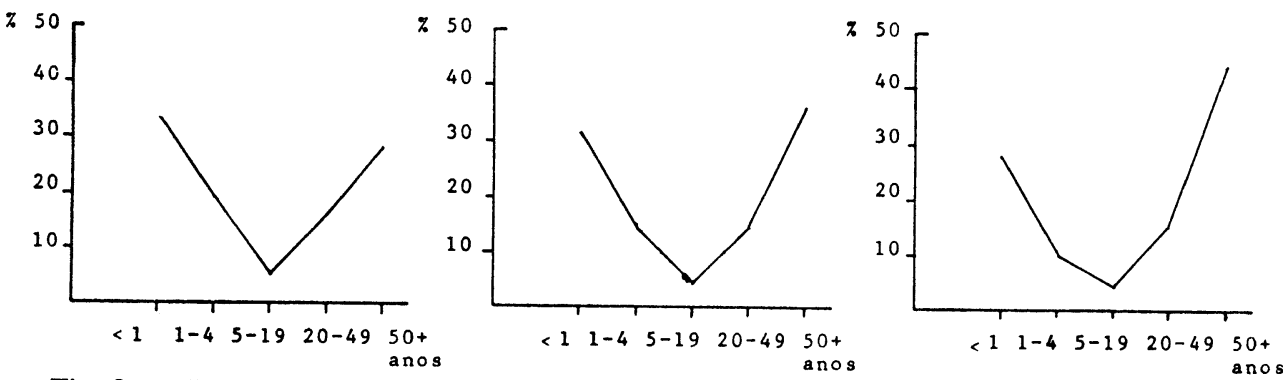

Fig. 2 - Curva de mortalidade proporcional para as regiões administrativas do de São Paulo. Dados médios para os períodos de 1950, 1960 e 1967.

Fonte dos dados brutos: Departamento de Estatística do Est. de São Paulo (DEE). 
C A M P I N A S
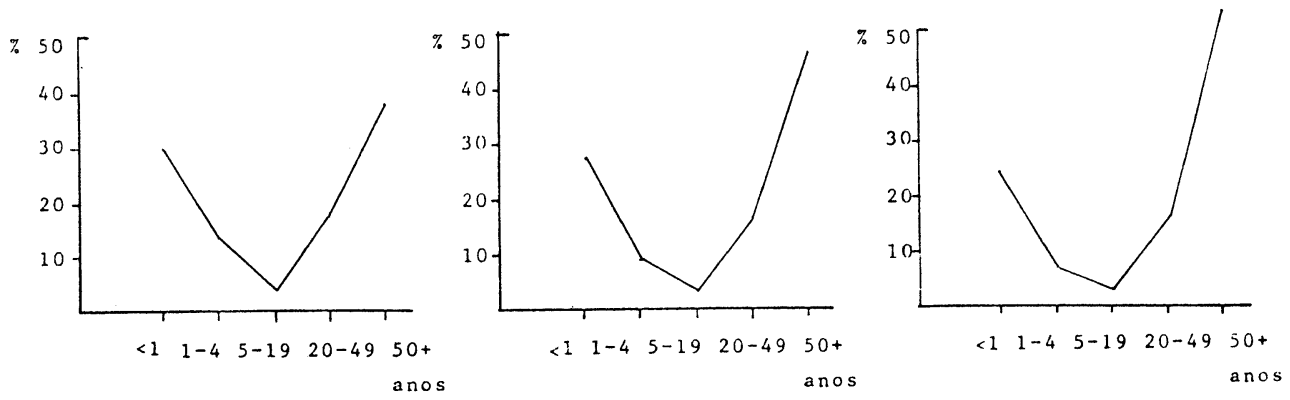

RIBEIRÃO PRETO
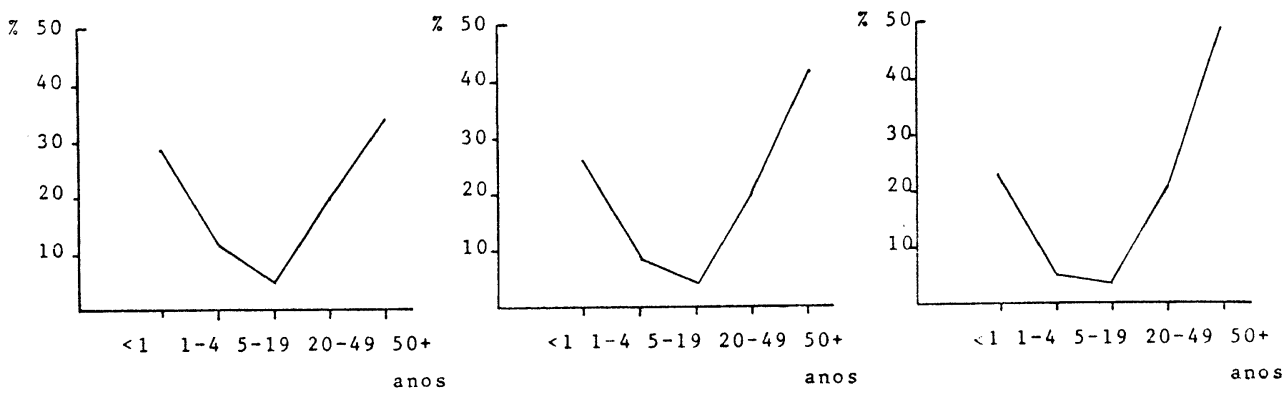

B A U R U

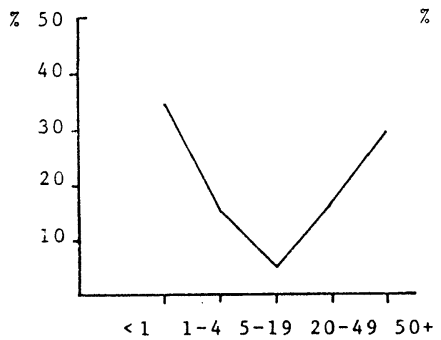

anos
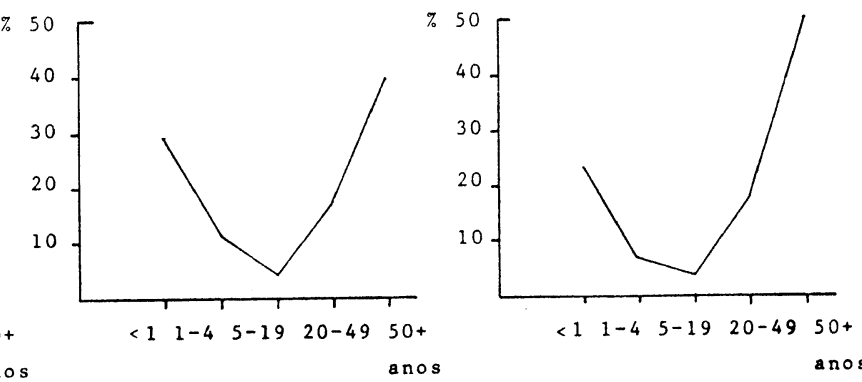

SÃO JOSE DO RIO PRETO
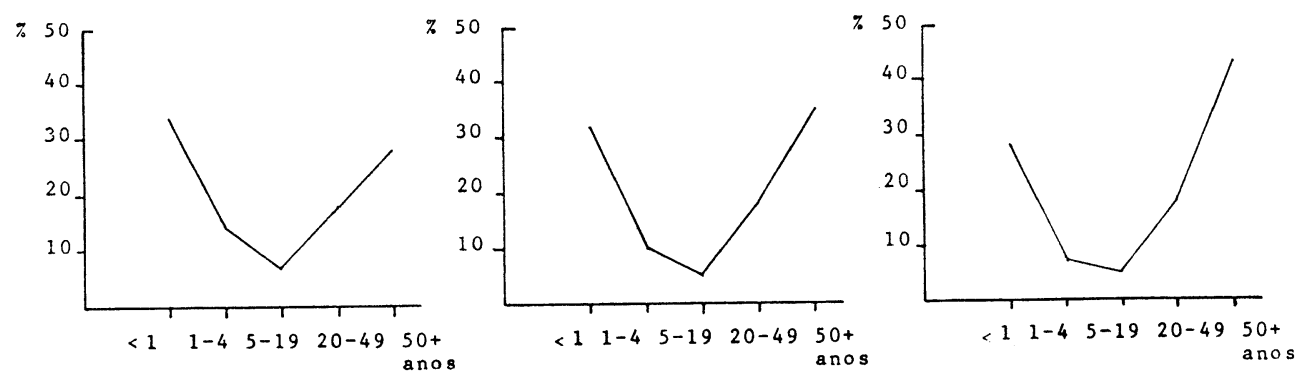

Fig. 3 - Curva de mortalidade proporcional para as regióes administrativas do Estado de São Paulo. Dados médios para os períodos de 1950, 1960 e 1967. Fonte dos dados brutos: DEE. 
A $\quad$ R $A$ A
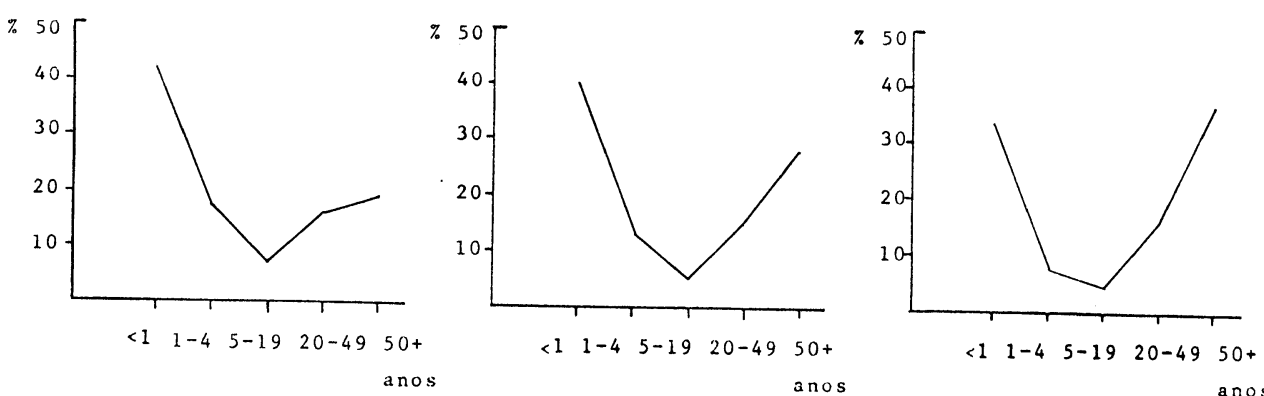

PRESIDENTE PRUDENTE
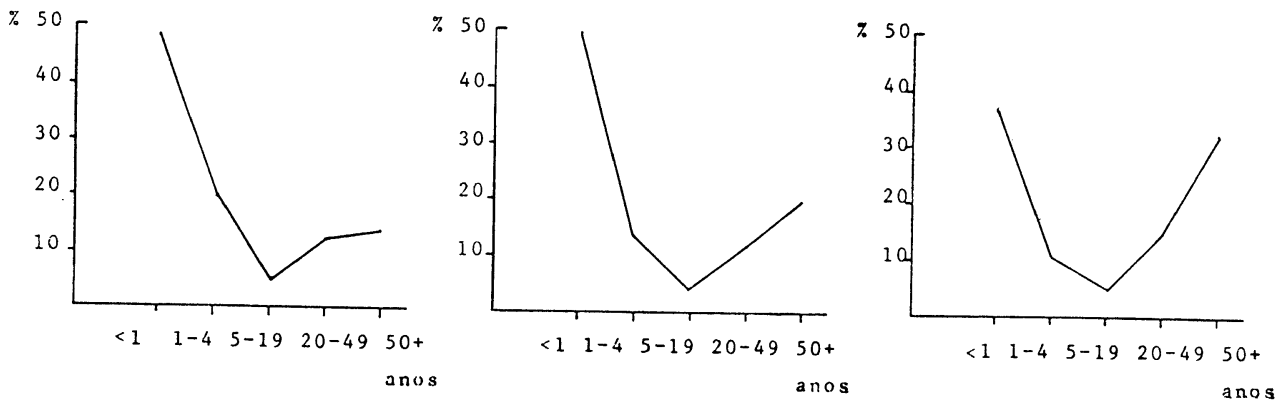

M A R I L I A
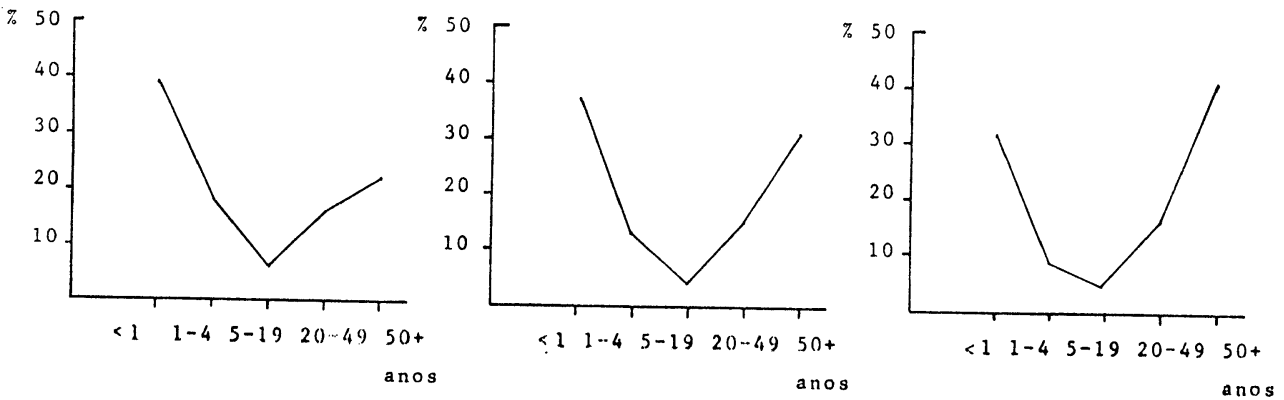

ETADO DE SÃO PAULO
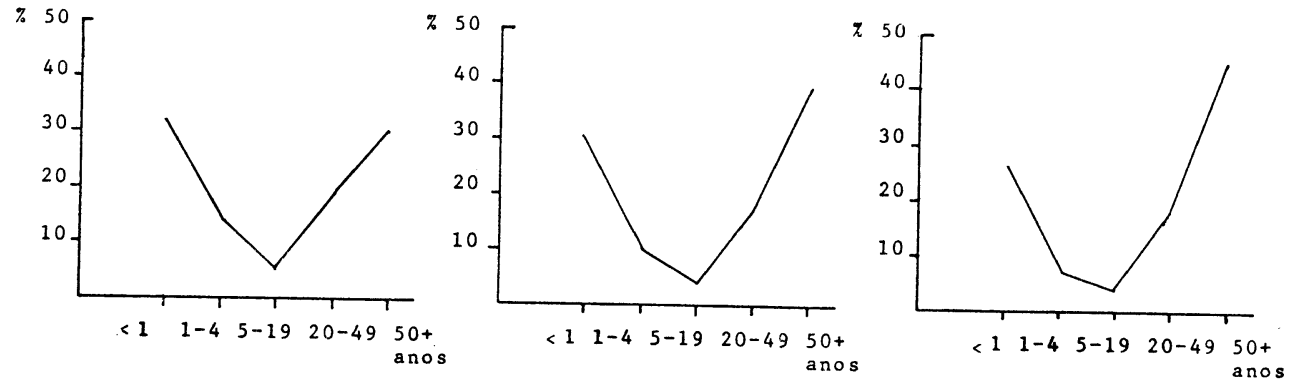

Fig. 4 - Curva de mortalidade proporcional para as regiões administrativas do Estado de São Paulo. Dados médios para os períodos de 1950, 1960 e 1967.

Fonte dos dados brutos: DEE. 


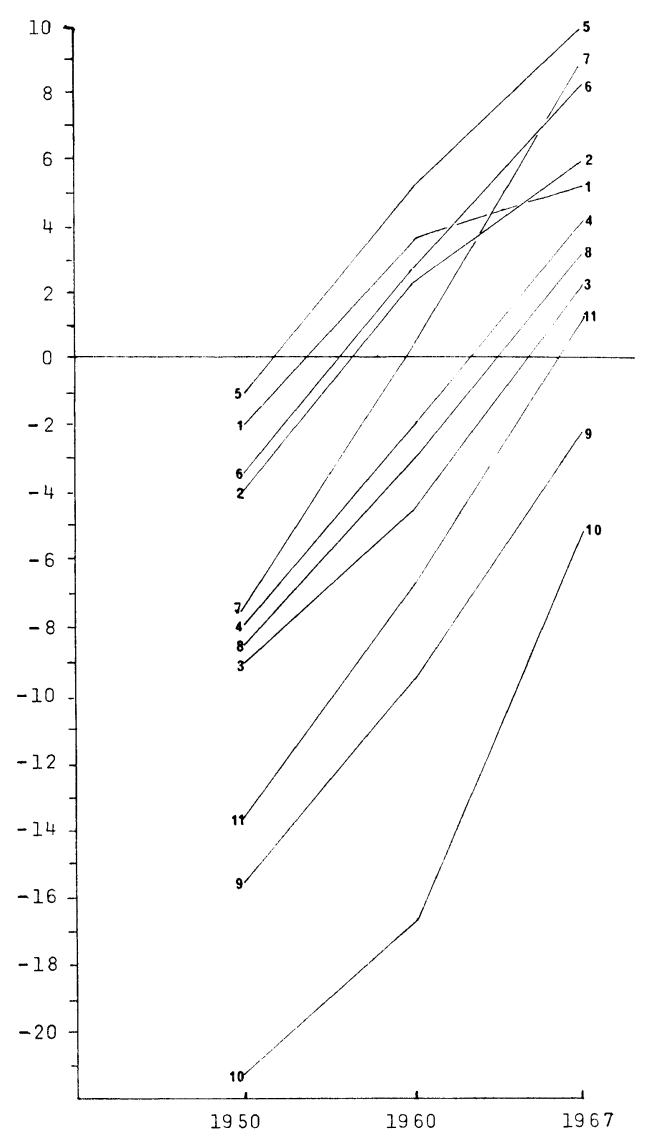

Fig. 5 - Evolução dar Curvas de Mortalidade Proporcional para as regióes administrativas nos períodos de 1950 , 1960 e 1967. - "dados quantitativos" - Estado de São Paulo. 